\title{
Psychotropic effects of aspirin, acetylsalicylate cobalt and acetylsalicylate zinc at various doses
}

\author{
Tatyana V. Yakovchyuk, Oksana V. Katiushyna*, Ivan I. Koreniuk, Denis R. Khusainov, \\ Tatyana V. Gamma
}

Department of Human and Animal Physiology and Biophysics, Faculty of Biology, National Tavrida Vernadskii University, Simferopol, Ukraine; ${ }^{*}$ Corresponding Author: oxakat@yandex.ru

Received 8 June 2012; revised 28 July 2012; accepted 11 September 2012

\begin{abstract}
For the first time it is shown that psychotropic action of acetylsalicylates at various doses is manifested as a nonmonotonic dependence having its peaks at therapeutic and ultra-low dose zones. It is discovered that development of effects of aspirin resembles that of acetylsalicylate zinc. Acetylsalicylate cobalt at extremely low doses zone showed the highest antidepressant activity, demonstrating toxicity at high doses. Generally, it is revealed that the use of aspirin and its salts at high doses range causes maximum psychotropic effects development, usually accompanied by side-effects. Therefore, aspirin, acetylsalicylate cobalt and zinc at extremely low doses are recommended for further study as psychotropic medications.
\end{abstract}

Keywords: Acetylsalicylic Acid; Acetylsalicylate Cobalt; Acetylsalicylate Zinc; Psychotropic

Action, Behavioral Tests; Ultra-Low Doses; Rats

\section{INTRODUCTION}

Today, the effectiveness of most pharmaceutical products is noted to rise as the dose is increased, in some cases reaching its maximum at high dosage rate. However, the administration of pharmaceutical products at therapeutic doses and at high doses, a fortiori, is usually accompanied by the development of a broad spectrum of side-effects [1]. Thus, for acetylsalicylic acid (aspirinAsp), along with the anti-inflammatory and analgesic action, the manifestation of psychotropic effects is ob- served at the upper limit of therapeutic dose (TD) [2]. Aspirin, in spite of having the great number of positive properties, also provokes stomach ulceration, negatively influences the cells of liver and of blood [3]. It should be noted that the spectrum of side-effects enlarges as the dose is increased. Taking into consideration the fact that many biologically active substances have been clinically indicated for administration at ultra-low doses (ULD) recently [4], aspirin at ULD is probable to preserve its therapeutic activity developing no side-effects. Due to the strictly individual nature of effect manifestation at ULD for every pharmaceutical product [5], this work is aimed to reveal the specificity of psychotropic effects of Asp and of its derivatives at various doses.

\section{MATERIALS AND METHODS}

The research was conducted at 250 white outbred male rats having 200 - $250 \mathrm{gr}$ of weight that were divided into 25 groups, 10 animals in each group. The studied substances (Asp and its derivatives - acetylsalicylate cobalt (ASC) and acetylsalicylate zinc (ASZ)) were diluted in normal saline and injected intraperitoneally (i.p.) $0.2 \mathrm{ml}$ at a time, at high doses (120 and $80 \mathrm{mg} / \mathrm{kg})$, at TD (40, $15 \mathrm{mg} / \mathrm{kg})$, "dead zone" dose $\left(40 \times 10^{-4} \mathrm{mg} / \mathrm{kg}\right)$ and at the three ULD $\left(40 \times 10^{-8}, 40 \times 10^{-10}, 40 \times 10^{-13} \mathrm{mg} / \mathrm{kg}\right)$. The animals of the control group were administered to the equivalent volume of normal saline solution. The TD and ULD zones are separated by the "dead zone" in dosage range $10^{-12}-10^{-8} \mathrm{~mol}$ where the psychotropic effects are absent (Table 1). The control point at $40 \times$ $10^{-4} \mathrm{mg} / \mathrm{kg}$ is taken to provide better illustration.

30 minutes after the i.p. injection we studied the behavioral response of the rats using Open Field test [6]

Table 1. The ratio of dose/concentration.

\begin{tabular}{|c|c|c|c|c|c|c|c|c|}
\hline & \multicolumn{2}{|c|}{ High } & \multicolumn{2}{|c|}{ Therapeutic } & \multirow{2}{*}{$\begin{array}{c}\text { Dead zone } \\
40 \times 10^{-4}\end{array}$} & \multicolumn{3}{|c|}{ Ultra-low } \\
\hline does, $\mathrm{mg} / \mathrm{kg}$ & 120 & 80 & 40 & 15 & & $40 \times 10^{-8}$ & $40 \times 10^{-10}$ & $40 \times 10^{-13}$ \\
\hline concentration, $\mathrm{M}$ & $3 \times 10^{-4}$ & $2 \times 10^{-4}$ & $10^{-4}$ & $3.8 \times 10^{-3}$ & $40 \times 10^{-8}$ & $10^{-12}$ & $10^{-14}$ & $10^{-17}$ \\
\hline
\end{tabular}


and Porsolt test [7].

The standard Open Field test is commonly used to assess locomotor, exploratory and anxiety-like behavior in laboratory animals (rats/mice). This test is particularly useful in evaluating the effects of anxiolytic and anxiogenic drugs, locomotor responses to drug and as well as behavioral responses to novelty. The Open Field area generally consists of an empty and bright square arena, surrounded by walls to prevent animal from escaping. The animal is usually place in the center of the arena and its behavior recorded for $3 \mathrm{~min}$. We are registered horizontal motor activity and research activity.

Forced swimming test (Porsolt's test). This is the most commonly used test for assessment of depressive status in animals. The experimental setup is a transparent cylinder filled with water of room temperature. A rat is placed in the water; its behavior is observed for $3 \mathrm{~min}$. The time of active swimming and the time of immobility (when a rat just weakly moves the paws to keep afloat) are registered. The latter is interpreted as "hope loss behavior" and is longer in animals with depressive-like status. This immobility time is decreased by antidepressants.

The reliability of disparity between the control group and the experimental group was estimated with the use of non-parametric U-criterion by Mann-Whitney (at $\mathrm{p} \leq$ $0.05, \mathrm{p} \leq 0.01)$. The data is represented in relative units (\%), values of the control group being taken for $100 \%$. During the experiments all the ethic norms were observed.

\section{RESULTS OF THE EXPERIMENTS}

The Figure 1 shows psychotropic effects of Asp in dependence on the dose in the Open Field test. It is obvious that Asp at high doses significantly raised the horizontal movement activity (HMA) and investigative activity (IA) which is the evidence of its marked anti-stress and anxiolytic action $(\mathrm{p} \leq 0.01)$ [6]. At the upper limit of TD of Asp (40 mg/kg) IA was decreased reliably and HMA - unreliably, that is, anxiogenic effect was produced instead of anxiolytic one, anti-stress action neutralized. At $40 \times 10^{-8} \mathrm{mg} / \mathrm{kg}$ Asp dose the effects of TD $(40 \mathrm{mg} / \mathrm{kg})$ were repeated. At lower doses $(40 \times$ $10^{-10}$ and $40 \times 10^{-13} \mathrm{mg} / \mathrm{kg}$ ) the anxiogenic action of Asp became obsolete, but anti-stress action was revealed against this background.

ASZ at high doses, as well as Asp, increased HMA and IA in the animals, which is the evidence of its anti-stress and anxiolytic action (Figure 2). The dose being decreased, ASZ shows the growth of its anxiolytic effect along with the reduction of anti-stress one. At the upper limit of TD $(40 \mathrm{mg} / \mathrm{kg})$ anxiolytic effect reaches its maximum, while anti-stress effect is not manifested at

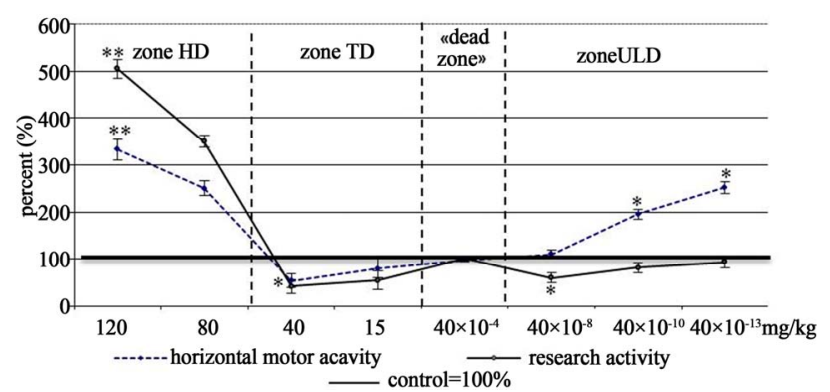

Note: HD - high dose; TD - therapeutic dose; ULD - ultra-low doses; $\mathrm{p}$-level of significance of disparity compared with control; ${ }^{*} \mathrm{p} \leq 0.05,{ }^{* *} \mathrm{p} \leq$ 0.01 .

Figure 1. The curves "dose-effect" of aspirin (open field test).

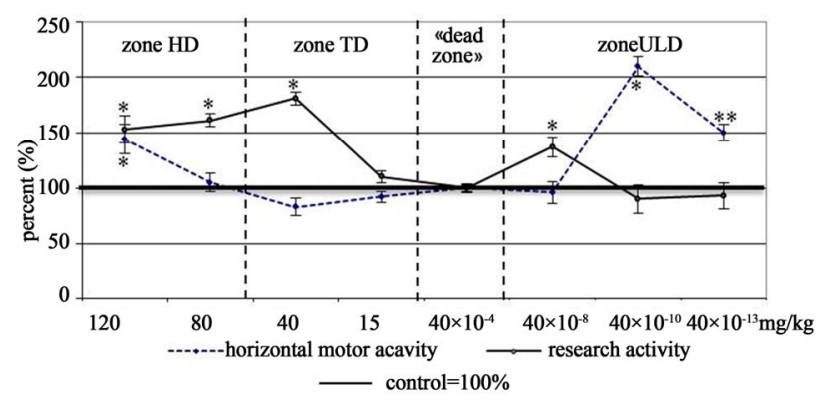

Note: (the same as at Figure 1).

Figure 2. The curves "dose-effect" of acetylsalicylate zinc (open field test).

TD Zone at all. Further decrease of the dose causes neutralization of psychotropic effects of ASZ. However, at $40 \times 10^{-8} \mathrm{mg} / \mathrm{kg}$ dose ASZ makes anxiolytic action, but it is less marked than that one at TD. At other ELD $(40 \times$ $10^{-10}$ and $40 \times 10^{-13} \mathrm{mg} / \mathrm{kg}$ ) anti-stress action of ASZ is well seen in the absence of anxiolytic effect.

ASC at high doses produced toxic effect. Under these conditions $70 \%$ of the sampling administered to 120 $\mathrm{mg} / \mathrm{kg}$ dose, and $40 \%$ of the sampling administered to 80 $\mathrm{mg} / \mathrm{kg}$ died. The surviving animals were excluded from further experiment. At the upper limit of TD anxiolytic action of ASC was manifested (Figure 3). The dose being decreased, the effect grew neutralized. At ULD Zone a certain regularity of effect development is discovered: the dose being decreased, anxiolytic action of ASC grows 2 times as much as that at TD, then at $40 \times 10^{-10}$ $\mathrm{mg} / \mathrm{kg}$ dose the effect is neutralized, to be manifested again at $40 \times 10^{-13} \mathrm{mg} / \mathrm{kg}$ dose, but with less intensity. It must be noted that anxiolytic effect of ASC at ULD is accompanied by anti-stress effect development, reaching its maximum at $40 \times 10^{-13} \mathrm{mg} / \mathrm{kg}$ dose.

Thus, judging by the results of the Open Field test we can see that the same way of psychotropic effects development is typical for both Asp and ASC. It must be noted that ASC has the most intensive anxiolytic action at ULD Zone $\left(40 \times 10^{-13} \mathrm{mg} / \mathrm{kg}\right)$ compared with Asp and 


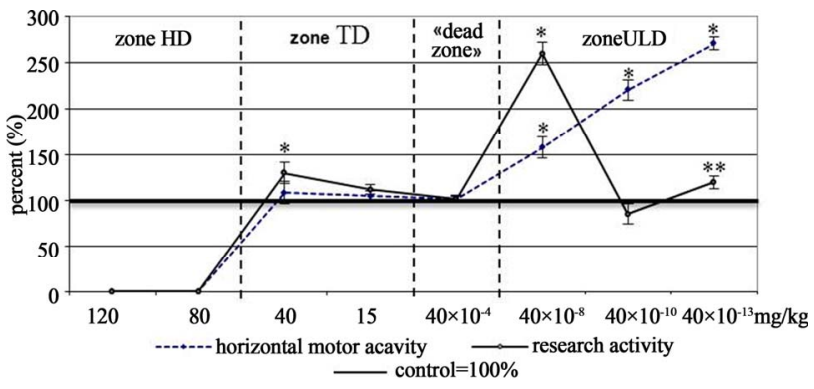

Note: (the same as at Figure 1).

Figure 3. The curves "dose-effect" of acetylsalicylate cobalt (open field test).

ASC, where substances preserve their biologically active properties, but side-effects are absent.

At Figure 4 we can see that in Porsolt test Asp at all doses extended the time of active swimming and reduced the time of immobility in experimental group rats as compared with the control group animals ( $p \leq 0,05)$, which is the evidence of its antidepressant action [8]. At $120 \mathrm{mg} / \mathrm{kg}$ dose Asp demonstrated development of the maximum antidepressant effect that decreased at TD (40 $\mathrm{mg} / \mathrm{kg}$ ). The dose being decreased, antidepressant effect was neutralized, to be manifested again at ULD Zone, repeating the TD effect.

ASZ at high doses showed antidepressant action. At the upper limit of TD it was reduced insignificantly (Figure 5). The decrease of the dose caused neutralization of the effect. At ULD Zone ASZ at $40 \times 10^{-8} \mathrm{mg} / \mathrm{kg}$ dose significantly raised the depression level in animals, which was manifested by reduction of active swimming time and, correspondingly, extension of immobility time. However, at $40 \times 10^{-10}$ and $40 \times 10^{-13} \mathrm{mg} / \mathrm{kg} \mathrm{ASZ} \mathrm{dem-}$ onstrated antidepressant effect. This fact indicates the inversion of substance operational sign at $40 \times 10^{-8}$ $\mathrm{mg} / \mathrm{kg}$ dose.

ASC at the upper limit of TD had antidepressant action. The dose brought down to $15 \mathrm{mg} / \mathrm{kg}$, this effect was neutralized, the same way as at the dead zone (Figure 6). At ELD Zone, all doses of ASC extended the time of active swimming and, correspondingly, reduced the time of immobility. This is the evidence of antidepressant effect of ASC at ULD. Consequently, of all the studied substances maximum antidepressant effect at ULD was demonstrated by ASC, compared with Asp and ASZ.

Thus, we have shown that in case of aspirinate administration at various doses their psychotropic action is manifested as a nonmonotonic dependence having its peaks at both TD and ELD zones. It must be noted that the development of psychotropic effect at ULD is accompanied by manifestation of the new properties. On summing up the data we discovered that ASC, causing acute toxicity at high doses, resulted in maximum intensity effects of all the tested compounds when used at ELD.

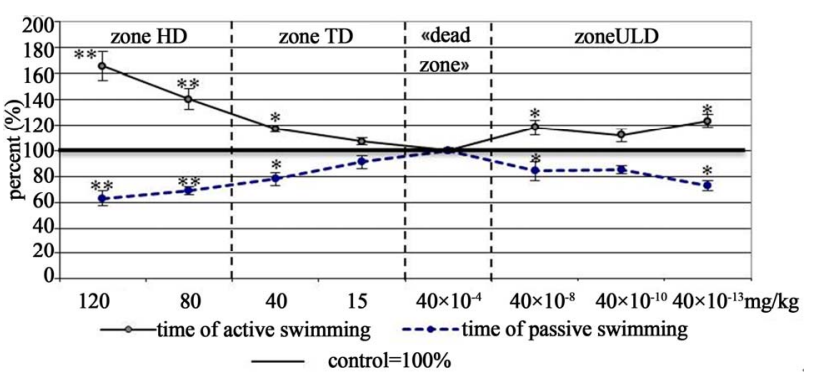

Note: (the same as at Figure 1).

Figure 4. The curves "dose-effect" of aspirin (porsolt test).

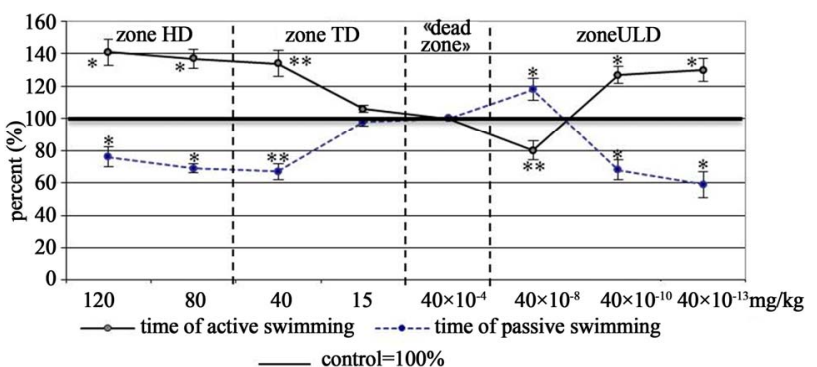

Note: (the same as at Figure 1).

Figure 5. The curves "dose-effect" of acetylsalicylate zinc (porsolt test).

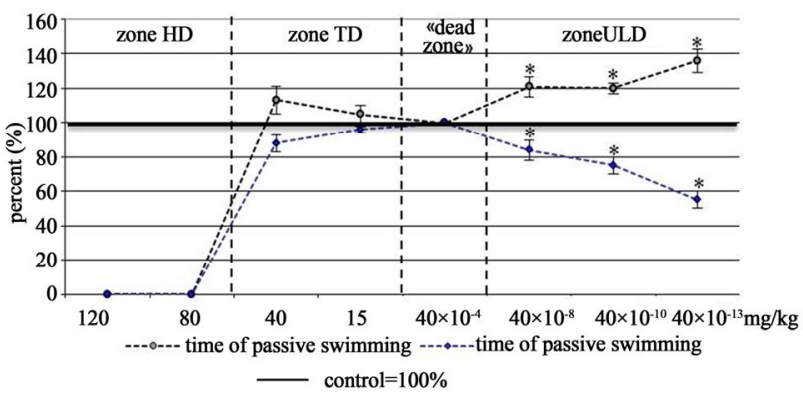

Note: (the same as at Figure 1)

Figure 6. The curves "dose-effect" of acetylsalicylate cobalt (porsolt test).

\section{DISCUSSION}

Generally, we have shown that administration of Asp and its salts at high doses causes maximum psychotropic effects to occur. However, the use of both Asp and its derivatives [9] at the studied dosage range is often accompanied by side-effects; moreover, for ASC at high doses the acute toxicity and, consequently, lethality is typical. So, the high doses of Asp must be administered with caution, while its newly synthesized derivatives require to be carefully studied as to having biological effectiveness at lower doses.

According to the results of this research, ULD zone has shown psychotropic action which repeats the effects of high doses and TD, at that approaching the maximum 
intensity at the lowest doses. The use of Asp at ULD offers a number of advantages: e.g., no side effects, a more pronounced effectiveness compared with $\mathrm{TD}$, etc. The results give us grounds for recommending the use of Asp at ULD in clinical testing as a psychotropic medication.

The absence of biological effects of Asp and its salts at $10^{-12}-10^{-8}$ range is explained by the "dead zone" existence. This phenomenon has been demonstrated in almost all experimental works. Formation of the "dead zone" is explained in literature by the hypothesis about the existence of the systems with negative feedback [10].

The material collected in the field of ULD study prevents us from skepticism towards ULD effects of biologically active substances, and, undoubtedly, the phenomenon of ULD is worth serious scientific studying. The presence of psychotropic effects of Asp and its salts at ULD range has its prerequisites justified by the data from the literature on the subject.

Today, Asp at low doses has indicated effectiveness in treatment of the vascular heart diseases, prevention of Alzheimer disease. Also, French scientists of Bordeaux II University and their Argentinean colleagues of Maimonides University, Buenos Aires have conducted a complex research on blood coagulability under aspirin influence at various doses [8]. First they studied the effects of the medication at therapeutic doses, after that - at low doses, then - to extremely low ones, and finally - to apparent concentrations. At that, they discovered an interesting phenomenon while studying the role of aspirin in blood clotting. The scientists decided to test the effectiveness of the medication made "after Hahnemann", that is, as a homeopathic preparation. It was found that "ultra-lowdose aspirin", or ULDA-H created antithrombotic effect by the mechanism other than that one at TD. While aspirin at TD knock out cyclooxygenase (COG-1), ULDA-H does not produce any effect on that, but it arrests COG-2 [11]. It is possible that Asp at ULD influences COG-2 getting to brain through hematoencephalic barrier. Moreover, it was shown that it is in brain where COG-2 is mainly contained, so it is only possible for non-steroidal anti-inflammatory drugs to reach anti-inflammatory effect in absence of side-effects on gastrointestinal tract and other tissues involved in COG-1 activity if they inhibit COG-2 activity electively [12].

However, no theory has been formulated yet in literature to explain to the fullest all the effects of extremely low doses of biologically active substances. Some efforts have been made to explain the observed effects by following hypotheses: concoction of the medical substance, existence of highly effective systems of receptor signaling transduction and enhancement, "adaptation" of receptors towards the action of extremely low doses [13], existence of supra-high-affinity receptors [14]. Also, analyzing the presented results it must be borne in mind that highly diluted solutions of Asp and its salts were prepared not by usual diluting method, but by the homeopathic potentiating method. Still, within the frame of this experiment the dosing frequency and the duration of aspirinate treatment were not taken into consideration depending on the potency and dose.

\section{CONCLUSION}

Thus, there are a number of advantages in use of Asp and its derivatives at ULD in mental illnesses treatment. Upon that, the research has a perspective of further analyzing distinctive features of course treatment with Asp and its salts and studying possible mechanisms of their action.

\section{REFERENCES}

[1] Barkagan, Z.S. and Kotovschikova, E.F. (2004) Comparative analysis of main and side effects of various forms of acetylsalicylic acid. Clinical Pharmacology and Therapy, 13, 1-4.

[2] Ketterer, M.W., Brymer, J., Rhoads, K., Kraft, P. and Lovallo, W.R. (1996) Is aspirin, as used for antithrombosis, an emotion-modulating, agent? Journal of Psychosomatic Research, 40, 53-58. doi:10.1016/0022-3999(95)00524-2

[3] Alekseyeva, L.I. (2009) Questions of use of non-steroidal anti-inflammatory drugs in rheumatology practice. Consilium Medicum Ukraina, 3, 3-10.

[4] Tochilkina, L.P. (2007) Phenomenon of extremely low doses, homeopathy and organophosphorus agent. Chemical and Biological Safety, 1, 4-14.

[5] Ashmarin, I.P., Karazeyeva, Y.P. and Lelekova, T.V. (1999) On the question of development of the problem of effectiveness of extremely low doses of biologically active compounds. Russian Journal of Chemistry, 37, 2128.

[6] Markel, A.L. (1981) To evaluation of general characteristics of rat behavior in the open field test. Journal of Higher Nervous Activity, 31, 301-307.

[7] Porsolt, R.D., Le Pinchon, M. and Jalfre, M. (1977) Depression: A new animal model sensitive to antidepressant treatments. Nature, 266, 730-732. doi:10.1038/266730a0

[8] Doutremepuich, C., Aguejouf O., Desplat, V. and Eizayaga, F.X. (2010) Aspirin therapy: An attempt to explain the events of prothrombotic complications after treatment discontinuation. Thrombosis and Haemostasis, 103, 171180. doi:10.1160/TH09-07-0506

[9] Yakovchyuk, T.V., Katyushyna, O.V., Husainov, D.R., Korenyuk, I.I., Kolotilova, O.I., Gamma, T.V. and Cheretayev, I.V. (2010) Influence of acetylsalicylic acid, cobalt and zinc aspirinates on rat behavior. Series: Biology, Chemistry, 23, 200-208.

[10] Sazanov, L.A. and Zaitsev, S.V. (1992) Effect of superlow doses (10-18-10-16 M) of biologically active substances: General trends, salient features, and possible mechanisms. 
Biochemistry, 57, 1443-1460.

[11] Zhang, J.J., Ding, E.L. and Song, Y.Q. (2006) Adverse effects of cyclooxygenase 2 inhibitors on renal and arrhythmia events. Clinician's Corner, 296, 1619-1632.

[12] Brenneis, C., Maier, T.J., Schmidt, R., et al. (2006) Inhibition of prostaglandin $\mathrm{E}_{2}$ synthesis by $\mathrm{SC}-560$ is independent of cyclooxygenase 1 inhibition. FASEB Journal, 20, 1352-1360. doi:10.1096/fj.05-5346com
[13] Chan, F.K. (2002) Celecoxib versus diclofenac and omeprazole in reducing the risk of recurrent ulcer bleeding in patients with arthritis. New England Journal of Medicine, 347, 2104-2110. doi:10.1056/NEJMoa021907

[14] Burlakova, Y.B., Kondratov, A.A. and Khudyakov, I.V. (1990) Exposure to chemical agents at very small doses on biological objects. Bulletin of the Academy of Science of the USSR, Series: Biology, 2, 183-194. 